Commun. Fac Sci. Univ. Ank. Ser A1 Math. Stat.

Volume 68, Number 2, Pages 1492 1505 (2019)

DOI: $10.31801 /$ cfsuasmas. 540642

ISSN 1303-5991 E-ISSN 2618-6470

http://communications.science.ankara.edu.tr/index.php?series=A1

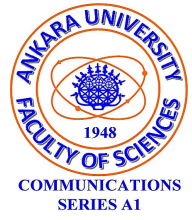

\title{
COEFFICIENT BOUNDS FOR A CERTAIN SUBCLASS OF ANALYTIC AND BI-UNIVALENT FUNCTIONS
}

\author{
NIZAMI MUSTAFA AND VEYSEL NEZIR
}

\begin{abstract}
In this paper, we introduce and investigate a new subclass of the analytic and bi-univalent functions in the open unit disk in the complex plane. For the functions belonging to this class, we obtain estimates on the first three coefficients in their Taylor-Maclaurin series expansion. Some interesting corollaries and applications of the results obtained here are also discussed.
\end{abstract}

\section{Introduction and Preliminaries}

Let $A$ denote the class of all complex-valued analytic functions in the open unit disk $U=\{z \in \mathbb{C}:|z|<1\}$ in the complex plane of the form

$$
f(z)=z+a_{2} z^{2}+a_{3} z^{3}+\cdots=z+\sum_{n=2}^{\infty} a_{n} z^{n}, z \in U .
$$

Furthermore, by $S$ we shall denote the class of all functions in $A$ which are univalent in $U$. Some of the important and well-investigated subclasses of $S$ include the class $S^{*}(\alpha)$ of starlike functions of order $\alpha$ and the class $C(\alpha)$ of convex functions of order $\alpha(\alpha \in[0,1))$.

By definition

$$
S^{*}(\alpha)=\left\{f \in S: \operatorname{Re}\left(\frac{z f^{\prime}(z)}{f(z)}\right)>\alpha, z \in U\right\}, \alpha \in[0,1)
$$

and

$$
C(\alpha)=\left\{f \in S: \operatorname{Re}\left(1+\frac{z f^{\prime}(z)}{f(z)}\right)>\alpha, z \in U\right\}, \alpha \in[0,1) .
$$

The above mentioned function classes have been recently investigated rather extensively in [10, 20, 26, 29] and the references therein.

It is well-known that every function $f \in S$ has an inverse $f^{-1}$, defined by $f^{-1}(f(z))=z, z \in U$ and $f\left(f^{-1}(w)\right)=w, w \in D=\left\{w \in \mathbb{C}:|w|<r_{0}(f)\right\}$, $r_{0}(f) \geq 1 / 4$ where $f^{-1}(w)=w-a_{2} w^{2}+\left(2 a_{2}^{2}-a_{3}\right) w^{3}-\left(5 a_{2}^{3}-5 a_{2} a_{3}+a_{4}\right) w^{4}+\cdots$.

Received by the editors: February 28, 2018, Accepted: October 08,2018.

2010 Mathematics Subject Classification. Primary 30C45, 30C50.

Key words and phrases. Univalent functions, analytic functions, bi-univalent functions. 
An analytic function $f$ is subordinate to an analytic function $\phi$, written $f(z) \prec$ $\phi(z)$, provided there is an analytic function $u: U \rightarrow U$ with $u(0)=0$ and $|u(z)|<1$ satisfying $f(z)=\phi(u(z))$ (see, for example, [14]).

Ma and Minda [12] unified various subclasses of starlike and convex functions for which either of the quantity $\frac{z f^{\prime}(z)}{f(z)}$ or $1+\frac{z f^{\prime \prime}(z)}{f^{\prime}(z)}$ is subordinate to a more superordinate function. For this purpose, they considered an analytic function $\phi$ with positive real part in $U$, with $\phi(0)=1, \phi^{\prime}(0)>0$ and $\phi$ maps $U$ onto a region starlike with respect to 1 and symmetric with respect to the real axis. The class of Ma-Minda starlike and Ma-Minda convex functions consists of functions $f \in A$ satisfying the subordination $\frac{z f^{\prime}(z)}{f(z)} \prec \phi(z)$ and $1+\frac{z f^{\prime \prime}(z)}{f^{\prime}(z)} \prec \phi(z)$, respectively. These classes denoted, respectively, by $S^{*}(\phi)$ and $C(\phi)$.

An analytic function $f \in S$ is said to be bi- starlike of Ma-Minda type or biconvex of Ma-Minda type if both $f$ and $f^{-1}$ are, respectively, Ma-Minda starlike or Ma-Minda convex functions. These classes are denoted, respectively, by $S_{\Sigma}^{*}(\phi)$ and $C_{\Sigma}(\phi)$. In the sequel, it is assumed that $\phi$ is an analytic function with positive real part in $U$, satisfying $\phi(0)=1, \phi^{\prime}(0)>0$ and $\phi(U)$ is starlike with respect to 1 and symmetric with respect to the real axis. Such a function has a series expansion of the following form:

$$
\phi(z)=1+b_{1} z+b_{2} z^{2}+b_{3} z^{3}+\cdots, b_{1}>0 .
$$

A function $f \in A$ is said to be bi-univalent in $U$ if both $f$ and $f^{-1}$ are univalent. Let $\Sigma$ denote the class of bi-univalent functions in $U$ given by (1.1).

Examples of functions in the class $\Sigma$ are

$$
\frac{z}{1-z}, \quad \ln \frac{1}{1-z}, \ln \sqrt{\frac{1+z}{1-z}} .
$$

However, the familiar Koebe function is not a member of $\Sigma$. Other common examples of functions in $A$ such as

$$
\frac{2 z-z^{2}}{2} \text { and } \frac{z}{1-z^{2}}
$$

are also not members of $\Sigma$.

Earlier, Brannan and Taha [3] introduced certain subclasses of bi-univalent function class $\Sigma$, namely bi-starlike function of order $\alpha$ denoted $S_{\Sigma}^{*}(\alpha)$ and bi-convex function of order $\alpha$ denoted $C_{\Sigma}(\alpha)$ corresponding to the function classes $S^{*}(\alpha)$ and $C(\alpha)$, respectively. Thus, following Brannan and Taha [3], a function $f \in \Sigma$ is in the classes $S_{\Sigma}^{*}(\alpha)$ and $C_{\Sigma}(\alpha)$, respectively, if each of the following conditions is satisfied:

$$
\operatorname{Re}\left(\frac{z f^{\prime}(z)}{f(z)}\right)>\alpha, z \in U, \operatorname{Re}\left(\frac{z g^{\prime}(w)}{g(w)}\right)>\alpha, w \in D
$$


and

$$
\operatorname{Re}\left(1+\frac{z f^{\prime}(z)}{f(z)}\right)>\alpha, z \in U, \operatorname{Re}\left(1+\frac{z g^{\prime}(w)}{g(w)}\right)>\alpha, w \in D .
$$

For each of the function classes $S_{\Sigma}^{*}(\alpha)$ and $C_{\Sigma}(\alpha)$, they found non-sharp estimates on the first two Taylor-Maclaurin coefficients $\left|a_{2}\right|$ and $\left|a_{3}\right|$.

Lewin [11] investigated bi-univalent function class $\Sigma$ and showed that $\left|a_{2}\right|<1.51$. Subsequently, Brannan and Clunie [2] conjectured that $\left|a_{2}\right|<\sqrt{2}$.

For a brief history and interesting examples of functions which are in the class $\Sigma$, together with various other properties of this bi-univalent function class, one can refer the work of Srivastava et al. 222 and references therein. In 22], Srivastava et al. reviewed the study of coefficient problems for bi-univalent functions. Also, various subclasses of bi-univalent function class were introduced and non-sharp estimates on the first two coefficients in the Taylor-Maclaurin series expansion (1.1) were found in several recent investigations (see, for example, [1, 4, 5, 6, 7, 8, 9, 13, 15, 19, 21, 23, 24, 25, 27, 28. Recently, Orhan et al. [17. reviewed the study of coefficient problems for the subclass $\operatorname{NP}_{\Sigma}^{\mu, \lambda}(\beta, h)$ of bi-univalent functions.

However, the problem to find the coefficient bounds on $\left|a_{n}\right|, n=3,4, \ldots$ for functions $f \in \Sigma$ is presumably still an open problem (see, for example [2, 11, 16]).

Inspired by the aforementioned works, we define a subclass of $\Sigma$ as follows.

Definition 1.1. A function $f \in \Sigma$ given by (1.1) is said to be in the class $M_{\Sigma}(\phi, \beta)$, $\beta \geq 0$, where $\phi$ is an analytic function given by $(1.2)$, if the following conditions are satisfied:

$$
\begin{gathered}
\left(\frac{z f^{\prime}(z)}{f(z)}\right)^{\beta}\left(1+\frac{z f^{\prime \prime}(z)}{f^{\prime}(z)}\right)^{1-\beta} \prec \phi(z), z \in U, \\
\left(\frac{z g^{\prime}(w)}{g(w)}\right)^{\beta}\left(1+\frac{z g^{\prime \prime}(w)}{g^{\prime}(w)}\right)^{1-\beta} \prec \phi(w), w \in D,
\end{gathered}
$$

where $g=f^{-1}$.

Remark 1.2. Taking $\beta=1$, we have $M_{\Sigma}(\phi, 1)=S_{\Sigma}^{*}(\phi)$; that is, $\frac{z f^{\prime}(z)}{f(z)} \prec \phi(z), z \in U$ and $\frac{z g^{\prime}(w)}{g(w)} \prec \phi(w), w \in D$

if and only if $f \in S_{\Sigma}^{*}(\phi)$, where $g=f^{-1}$.

Remark 1.3. Taking $\beta=0$, we have $M_{\Sigma}(\phi, 0)=C_{\Sigma}(\phi)$; that is,

$1+\frac{z f^{\prime}(z)}{f(z)} \prec \phi(z), z \in U$ and $1+\frac{z g^{\prime}(w)}{g(w)} \prec \phi(w), w \in D$ if and only if $f \in C_{\Sigma}(\phi)$, where $g=f^{-1}$.

Remark 1.4. These classes $S_{\Sigma}^{*}(\phi)$ and $C_{\Sigma}(\phi)$ were investigated by Ma and Minda 12.

The object of this paper is to introduce a new subclass $M_{\Sigma}(\phi, \beta)$ of the function class $\Sigma$ that is wider (respect to $\beta$ ) to the subclasses examined so far and to find 
estimates on the first three Taylor-Maclaurin coefficients $\left|a_{2}\right|,\left|a_{3}\right|$ and $\left|a_{4}\right|$ for the functions in this class.

To prove our main results, we have to recall the following well-known Lemma [18.

Lemma 1.5. Let $\mathrm{P}$ be the class of all analytic functions $p(z)$ of the form

$$
p(z)=1+p_{1} z+p_{2} z^{2}+\cdots=1+\sum_{n=1}^{\infty} p_{n} z^{n}
$$

satisfying $\operatorname{Re}(p(z))>0, z \in U$ and $p(0)=1$. Then,

$$
\begin{gathered}
2 p_{2}=p_{1}^{2}+\left(4-p_{1}^{2}\right) x, \\
4 p_{3}=p_{1}^{3}+2\left(4-p_{1}^{2}\right) p_{1} x-\left(4-p_{1}^{2}\right) p_{1} x^{2}+2\left(4-p_{1}^{2}\right)\left(1-|x|^{2}\right) z,
\end{gathered}
$$

for some $x, z$ with $|x| \leq 1,|z| \leq 1$ and $p_{1} \in[0,2]$.

\section{Coefficient bounds for the function Class $M_{\Sigma}(\phi, \beta)$}

In this section, we will try to find the estimates on the coefficients $\left|a_{2}\right|,\left|a_{3}\right|$ and $\left|a_{4}\right|$ for the functions in the class $M_{\Sigma}(\phi, \beta)$.

Theorem 2.1. Let the function $f(z)$ given by (1.1) be in the class $M_{\Sigma}(\phi, \beta), \beta \in$ $[0,1]$, where $\phi$ is an analytic function given by $(1.2)$. Then,

$$
\left|a_{2}\right| \leq \frac{b_{1}}{2-\beta},\left|a_{3}\right| \leq \begin{cases}\frac{b_{1}^{2}}{(2-\beta)^{2}}, & \text { if } b_{1} \leq \frac{(2-\beta)^{2}}{2(3-2 \beta)}, \\ \frac{b_{1}}{2(3-2 \beta)}, & \text { if } b_{1}>\frac{(2-\beta)^{2}}{2(3-2 \beta)}\end{cases}
$$

and

$$
\left|a_{4}\right| \leq \min \left\{\frac{\left|b_{1}^{3} \varphi(\beta)-6(2-\beta)^{3} \Lambda\right|+6(2-\beta)^{3}\left|2 b_{2}-b_{1}\right|}{18(2-\beta)^{3}(4-3 \beta)}, \frac{b_{1}}{3(4-3 \beta)}\right\},
$$

where $\varphi(\beta)=\beta^{3}-3 \beta^{2}-46 \beta+60>0$ and $\Lambda=\Lambda\left(b_{1}, b_{2}, b_{3}\right)=b_{1}-2 b_{2}+b_{3}$.

Proof. Let $f \in M_{\Sigma}(\phi, \beta), \beta \in[0,1]$, where $\phi$ is an analytic function given by 1.2 and $g=f^{-1}$. Then, there are analytic functions $u: U \rightarrow U, v: D \rightarrow D$ with $u(0)=0=v(0),|u(z)|<1,|v(w)|<1$ and satisfying

$$
\begin{aligned}
& \left(\frac{z f^{\prime}(z)}{f(z)}\right)^{\beta}\left(1+\frac{z f^{\prime \prime}(z)}{f^{\prime}(z)}\right)^{1-\beta}=\phi(u(z)) \\
& \text { and }\left(\frac{w g^{\prime}(w)}{g(w)}\right)^{\beta}\left(1+\frac{w g^{\prime \prime}(w)}{g^{\prime}(w)}\right)^{1-\beta}=\phi(v(w)) .
\end{aligned}
$$

Let us define the functions $p(z)$ and $q(w)$ by

$p(z)=\frac{1+u(z)}{1-u(z)}=1+\sum_{n=1}^{\infty} p_{n} z^{n}, z \in U$ and $q(w)=\frac{1+v(w)}{1-v(w)}=1+\sum_{n=1}^{\infty} q_{n} w^{n}, w \in D$.

It follows that 


$$
u(z)=\frac{p(z)-1}{p(z)+1}=\frac{1}{2}\left\{p_{1} z+\left[p_{2}-\frac{p_{1}^{2}}{2}\right] z^{2}+\left[p_{3}-p_{1} p_{2}+\frac{p_{1}^{3}}{4}\right] z^{3}+\cdots\right\}
$$

and

$$
v(w)=\frac{q(w)-1}{q(w)+1}=\frac{1}{2}\left\{q_{1} w+\left[q_{2}-\frac{q_{1}^{2}}{2}\right] w^{2}+\left[q_{3}-q_{1} q_{2}+\frac{q_{1}^{3}}{4}\right] w^{3}+\cdots\right\} .
$$

Using 2.2 and 2.3 in 1.2 , we can easily write

$$
\begin{aligned}
\phi(u(z))= & 1+\frac{b_{1} p_{1}}{2} z+\left[\frac{b_{1}}{2}\left(p_{2}-\frac{p_{1}^{2}}{2}\right)+\frac{1}{4} b_{2} p_{1}^{2}\right] z^{2} \\
& +\left[\frac{b_{1}}{2}\left(p_{3}-p_{1} p_{2}+\frac{p_{1}^{3}}{4}\right)+\frac{b_{2} p_{1}}{2}\left(p_{2}-\frac{p_{1}^{2}}{2}\right)+\frac{b_{3} p_{1}^{3}}{8}\right] z^{3}+\cdots
\end{aligned}
$$

and

$$
\begin{aligned}
\phi(v(w))= & 1+\frac{b_{1} q_{1}}{2} w+\left[\frac{b_{1}}{2}\left(q_{2}-\frac{q_{1}^{2}}{2}\right)+\frac{1}{4} b_{2} q_{1}^{2}\right] w^{2} \\
& +\left[\frac{b_{1}}{2}\left(q_{3}-q_{1} q_{2}+\frac{q_{1}^{3}}{4}\right)+\frac{b_{2} q_{1}}{2}\left(q_{2}-\frac{q_{1}^{2}}{2}\right)+\frac{b_{3} q_{1}^{3}}{8}\right] w^{3}+\cdots
\end{aligned}
$$

Also, using (2.4) and 2.5) in 2.1) and equating the coefficients, we get

$$
\begin{gathered}
(2-\beta) a_{2}=\frac{b_{1} p_{1}}{2}, \\
2(3-2 \beta) a_{3}+\frac{1}{2}\left(\beta^{2}+5 \beta-8\right) a_{2}^{2}=\frac{b_{1}}{2}\left(p_{2}-\frac{p_{1}^{2}}{2}\right)+\frac{1}{4} b_{2} p_{1}^{2}, \\
3(4-3 \beta) a_{4}+\left(4 \beta^{2}+11 \beta-18\right) a_{2} a_{3}-\frac{1}{6}\left(\beta^{3}+21 \beta^{2}+20 \beta-48\right) a_{2}^{3} \\
=\frac{b_{1}}{2}\left(p_{3}-p_{1} p_{2}+\frac{p_{1}^{3}}{4}\right)+\frac{b_{2} p_{1}}{2}\left(p_{2}-\frac{p_{1}^{2}}{2}\right)+\frac{b_{3} p_{1}^{3}}{8}
\end{gathered}
$$

and

$$
\begin{gathered}
-(2-\beta) a_{2}=\frac{b_{1} q_{1}}{2} \\
-2(3-2 \beta) a_{3}+\frac{1}{2}\left(\beta^{2}-11 \beta+16\right) a_{2}^{2}=\frac{b_{1}}{2}\left(q_{2}-\frac{q_{1}^{2}}{2}\right)+\frac{1}{4} b_{2} q_{1}^{2}, \\
-3(4-3 \beta) a_{4}+\left(4 \beta^{2}-34 \beta+42\right) a_{2} a_{3}+\frac{1}{6}\left(\beta^{3}-27 \beta^{2}+158 \beta-192\right) a_{2}^{3} \\
=\frac{b_{1}}{2}\left(q_{3}-q_{1} q_{2}+\frac{p_{1}^{3}}{4}\right)+\frac{b_{2} q_{1}}{2}\left(q_{2}-\frac{q_{1}^{2}}{2}\right)+\frac{b_{3} q_{1}^{3}}{8}
\end{gathered}
$$

From 2.6 and $(2.9)$, we have

which is equivalent to

$$
a_{2}=\frac{b_{1} p_{1}}{2(2-\beta)}=\frac{-b_{1} q_{1}}{2(2-\beta)}
$$

$$
p_{1}=-q_{1} .
$$

By subtracting from (2.7) to 2.10 and considering 2.12 and 2.13), we can easily obtain 


$$
a_{3}=\frac{b_{1}^{2} p_{1}^{2}}{4(2-\beta)^{2}}+\frac{b_{1}\left(p_{2}-q_{2}\right)}{8(3-2 \beta)} .
$$

On the other hand, subtracting (2.11) from 2.8 and considering 2.12 and 2.14, we get

$$
\begin{aligned}
a_{4}= & \frac{b_{1}^{3} p_{1}^{3} \varphi(\beta)}{144(2-\beta)^{3}(4-3 \beta)}+\frac{5 b_{1}^{2} p_{1}\left(p_{2}-q_{2}\right)}{32(2-\beta)(3-2 \beta)}+\frac{b_{1}\left(p_{3}-q_{3}\right)}{12(4-3 \beta)} \\
& +\frac{\left(b_{2}-b_{1}\right) p_{1}\left(p_{2}+q_{2}\right)}{12(4-3 \beta)}-\frac{p_{1}^{3} \Lambda}{24(4-3 \beta)},
\end{aligned}
$$

where $\varphi(\beta)=\beta^{3}-3 \beta^{2}-46 \beta+60>0$ and $\Lambda=\Lambda\left(b_{1}, b_{2}, b_{3}\right)=b_{1}-2 b_{2}+b_{3}$.

Since $p_{1}=-q_{1}$, according to Lemma 1.5 we write

$$
p_{2}-q_{2}=\frac{4-p_{1}^{2}}{2}(x-y), p_{2}+q_{2}=p_{1}^{2}+\frac{4-p_{1}^{2}}{2}(x+y)
$$

and

$$
\begin{aligned}
p_{3}-q_{3}= & \frac{p_{1}^{3}}{2}+\frac{p_{1}\left(4-p_{1}^{2}\right)}{2}(x+y)-\frac{p_{1}\left(4-p_{1}^{2}\right)}{4}\left(x^{2}+y^{2}\right) \\
& +\frac{4-p_{1}^{2}}{2}\left[\left(1-|x|^{2}\right) z-\left(1-|y|^{2}\right) w\right] .
\end{aligned}
$$

for some $x, y, z, w$ with $|x| \leq 1,|y| \leq 1,|z| \leq 1,|w| \leq 1$. In this case, since $p_{1} \in[0,2]$, we may assume without any restriction that $t \in[0,2]$, where $t=\left|p_{1}\right|$. Hence, we find from $(2.12)$ that

$$
\left|a_{2}\right| \leq \frac{b_{1}}{2-\beta} .
$$

Substituting the first expression 2.16 in 2.14, we obtain

$$
a_{3}=\frac{b_{1}^{2} p_{1}^{2}}{4(2-\beta)^{2}}+\frac{b_{1}\left(4-p_{1}^{2}\right)}{16(3-2 \beta)}(x-y) .
$$
have

Applying triangle inequality on the last equation and taking $\xi=|x|, \eta=|y|$, we

where

$$
\left|a_{3}\right| \leq c_{1}(t)+c_{2}(t)(\xi+\eta)
$$

$$
c_{1}(t)=\frac{b_{1}^{2} t^{2}}{4(2-\beta)^{2}} \geq 0, c_{2}(t)=\frac{b_{1}\left(4-t^{2}\right)}{16(3-2 \beta)} \geq 0, t \in[0,2] .
$$

Let us define the function $F: \mathbb{R}^{3} \rightarrow \mathbb{R}$ as follows:

$$
F(\xi, \eta, t)=c_{1}(t)+c_{2}(t)(\xi+\eta), \quad(\xi, \eta) \in \Omega, t \in[0,2],
$$

where $\Omega=\{(\xi, \eta): \xi, \eta \in[0,1]\}$.

From 2.18) and (2.19), we can write 


$$
\left|a_{3}\right| \leq \min \{\max \{F(\xi, \eta, t):(\xi, \eta) \in \Omega\}: t \in[0,2]\} .
$$

We can easily show that

$$
\max \{F(\xi, \eta, t):(\xi, \eta) \in \Omega\}=F(1,1, t)=c_{1}(t)+2 c_{2}(t), t \in[0,2] .
$$

Now, let us define the function $H: \mathbb{R} \rightarrow \mathbb{R}$ as follows:

$$
H(t)=c_{1}(t)+2 c_{2}(t), t \in[0,2] .
$$

Substituting the value of $c_{1}(t)$ and $c_{2}(t)$ in the above function, we have

$$
H(t)=\frac{b_{1}}{2(3-2 \beta)}+\frac{\Delta\left(\beta, b_{1}\right)}{8(3-2 \beta)(2-\beta)^{2}} t^{2},
$$

where $\Delta\left(\beta, b_{1}\right)=2(3-2 \beta) b_{1}^{2}-(2-\beta)^{2} b_{1}$.

Differentiating both sides of 2.22 , we get

$$
H^{\prime}(t)=\frac{\Delta\left(\beta, b_{1}\right)}{4(3-2 \beta)(2-\beta)^{2}} t .
$$

It is clear that $H^{\prime}(t) \leq 0$ if $0<b_{1} \leq \frac{(2-\beta)^{2}}{2(3-2 \beta)}$; that is, $H(t)$ is a decreasing function. Therefore,

$$
\min \{H(t): t \in[0,2]\}=H(2)=\frac{b_{1}^{2}}{(2-\beta)^{2}} .
$$

Let $b_{1}>\frac{(2-\beta)^{2}}{2(3-2 \beta)}$, then $H^{\prime}(t)>0$, so $H(t)$ is a strictly increasing function.

Therefore,

$$
\min \{H(t): t \in[0,2]\}=H(0)=\frac{b_{1}}{2(3-2 \beta)} .
$$

Consequently, from (2.21)-(2.24) and (2.20), we have

$$
\left|a_{3}\right| \leq \begin{cases}\frac{b_{1}^{2}}{(2-\beta)^{2}}, & \text { if } b_{1} \leq \frac{(2-\beta)^{2}}{2(3-2 \beta)}, \\ \frac{b_{1}}{2(3-2 \beta)}, & \text { if } b_{1}>\frac{(2-\beta)^{2}}{2(3-2 \beta)} .\end{cases}
$$

Substituting the expressions (2.16) and 2.17) in 2.15), we obtain

$$
\begin{aligned}
a_{4}= & \frac{b_{1}\left(4-p_{1}^{2}\right)}{24(4-3 \beta)}\left[\left(1-|x|^{2}\right) z-\left(1-|y|^{2}\right) w\right]-\frac{b_{1}\left(4-p_{1}^{2}\right) p_{1}}{48(4-3 \beta)}\left(x^{2}+y^{2}\right) \\
& +\frac{b_{2}\left(4-p_{1}^{2}\right) p_{1}}{24(4-3 \beta)}(x+y)+\frac{5 b_{1}^{2}\left(4-p_{1}^{2}\right) p_{1}}{64(2-\beta)(3-2 \beta)}(x-y) \\
& +\frac{b_{1}^{3} \varphi(\beta)-6(2-\beta)^{3} \Lambda+6(2-\beta)^{3}\left(2 b_{2}-b_{1}\right)}{144(2-\beta)^{3}(4-3 \beta)} p_{1}^{3} .
\end{aligned}
$$

Applying triangle inequality on the last equation, we have 


$$
\left|a_{4}\right| \leq d_{1}(t)\left(\xi^{2}+\eta^{2}\right)+d_{2}(t)(\xi+\eta)+d_{3}(t)
$$

where

$$
\begin{gathered}
d_{1}(t)=\frac{b_{1}\left(4-t^{2}\right)(t-2)}{48(4-3 \beta)} \leq 0, \\
d_{2}(t)=\frac{\left(4-t^{2}\right) t\left[8\left|b_{2}\right|(2-\beta)(3-2 \beta)+15 b_{1}^{2}(4-3 \beta)\right]}{192(2-\beta)(3-2 \beta)(4-3 \beta)} \geq 0, \\
d_{3}(t)=\frac{\left|b_{1}^{3} \varphi(\beta)-6(2-\beta)^{3} \Lambda\right|+6(2-\beta)^{3}\left|2 b_{2}-b_{1}\right|}{144(2-\beta)^{3}(4-3 \beta)} t^{3}+\frac{b_{1}\left(4-t^{2}\right)}{12(4-3 \beta)} \geq 0 .
\end{gathered}
$$

Let us define the function $G: \mathbb{R}^{3} \rightarrow \mathbb{R}$ as follows:

$$
G(\xi, \eta, t)=d_{1}(t)\left(\xi^{2}+\eta^{2}\right)+d_{2}(t)(\xi+\eta)+d_{3}(t),(\xi, \eta) \in \Omega, t \in[0,2] .
$$

From 2.26) and (2.27), we can write

$$
\left|a_{4}\right| \leq \min \{\max \{G(\xi, \eta, t):(\xi, \eta) \in \Omega\}: t \in[0,2]\} .
$$

Firstly, we need investigate maximum of the function $G(\xi, \eta, t)$ on the closed square $\Omega$ for each $t \in[0,2]$. Since the coefficients of the function $G(\xi, \eta, t)$ is dependent to variable $t$, we must investigate this maximum respect to $t$ taking into account these cases: $t=0, t \in(0,2)$ and $t=2$.

For $t=0$ we have

$$
G_{0}(\xi, \eta)=G(\xi, \eta, 0)=\frac{-b_{1}}{6(4-3 \beta)}\left(\xi^{2}+\eta^{2}\right)+\frac{b_{1}}{3(4-3 \beta)},(\xi, \eta) \in \Omega .
$$

We can easily show that the maximum of the function $G_{0}(\xi, \eta)$ occurs at $(\xi, \eta)=$ $(0,0)$, and

$$
\max \left\{G_{0}(\xi, \eta):(\xi, \eta) \in \Omega\right\}=G_{0}(0,0)=\frac{b_{1}}{3(4-3 \beta)} .
$$

In the case $t \in(0,2)$, by simple differentiation, we get

$$
\begin{aligned}
& G_{\xi}^{\prime}(\xi, \eta, t)=2 d_{1}(t) \xi+d_{2}(t), G_{\eta}^{\prime}(\xi, \eta, t)=2 d_{1}(t) \eta+d_{2}(t), \\
& G_{\xi \xi}^{\prime \prime}(\xi, \eta, t)=G_{\eta \eta}^{\prime \prime}(\xi, \eta, t)=2 d_{1}(t), G_{\xi \eta}^{\prime \prime}(\xi, \eta, t)=G_{\eta \xi}^{\prime \prime}(\xi, \eta, t)=0 .
\end{aligned}
$$

From the first and second equations above, we see that $\left(\xi_{0}, \eta_{0}\right)$, where $\xi_{0}=\eta_{0}=$ $\frac{-d_{2}(t)}{2 d_{1}(t)}$, is critical and likely a extremal point for of the function $G(\xi, \eta, t)$.

Since

$$
\Delta\left(\xi_{0}, \eta_{0}\right)=G_{\xi \xi}^{\prime \prime}\left(\xi_{0}, \eta_{0}, t\right) G_{\eta \eta}^{\prime \prime}\left(\xi_{0}, \eta_{0}, t\right)-\left[G_{\xi \xi}^{\prime \prime}\left(\xi_{0}, \eta_{0}, t\right)\right]^{2}=4 d_{1}^{2}(t)>0
$$


and $G_{\xi \xi}^{\prime \prime}(\xi, \eta, t)=G_{\eta \eta}^{\prime \prime}(\xi, \eta, t)=2 d_{1}(t)<0,\left(\xi_{0}, \eta_{0}\right)$ is a likely maximum point for the function $G(\xi, \eta, t)$. But, it is clear that $\left(\xi_{0}, \eta_{0}\right)$ is not a local maximum point if $\frac{-d_{2}(t)}{2 d_{1}(t)}>0$; that is if $\left(\xi_{0}, \eta_{0}\right) \notin \Omega$. We assume that $\left(\xi_{0}, \eta_{0}\right) \in \Omega$. In this case $\left(\xi_{0}, \eta_{0}\right)$ is a local maximum point for the function $G(\xi, \eta, t)$.

Therefore,

$$
\max \{G(\xi, \eta, t):(\xi, \eta) \in \Omega\}=G\left(\xi_{0}, \eta_{0}, t\right)=d_{3}(t)-\frac{-d_{2}^{2}(t)}{2 d_{1}(t)} .
$$

Let us define the function $h: \mathbb{R} \rightarrow \mathbb{R}$ by

$$
h(t)=d_{3}(t)-\frac{-d_{2}^{2}(t)}{2 d_{1}(t)}, t \in(0,2) .
$$

Substituting the value $d_{1}(t), d_{2}(t)$ and $d_{3}(t)$ in the above function, we have

where

$$
h(t)=h_{1} t^{3}+h_{2} t^{2}+h_{3}, t \in(0,2),
$$

$$
\begin{gathered}
h_{1}=\frac{\left|b_{1}^{3} \varphi(\beta)-6(2-\beta)^{3} \Lambda\right|+6(2-\beta)^{3}\left|2 b_{2}-b_{1}\right|}{144(2-\beta)^{3}(4-3 \beta)} \\
+\frac{\left[8\left|b_{2}\right|(2-\beta)(3-2 \beta)+15 b_{1}^{2}(4-3 \beta)\right]^{2}}{1536(2-\beta)^{2}(3-2 \beta)^{2}(4-3 \beta) b_{1}}>0, \\
h_{2}=\frac{\left[8\left|b_{2}\right|(2-\beta)(3-2 \beta)+15 b_{1}^{2}(4-3 \beta)\right]^{2}}{768(2-\beta)^{2}(3-2 \beta)^{2}(4-3 \beta) b_{1}}-\frac{b_{1}}{12(4-3 \beta)}, \\
h_{3}=\frac{b_{1}}{3(4-3 \beta)}>0 .
\end{gathered}
$$

Also, we consider the function $\bar{h}: \mathbb{R} \rightarrow \mathbb{R}$ as follows:

$$
\bar{h}(t)=h_{1} t^{3}+\bar{h}_{2} t^{2}+h_{3}, t \in(0,2),
$$

where

$$
\bar{h}_{2}=h_{2}+\frac{b_{1}}{12(4-3 \beta)}=\frac{\left[8\left|b_{2}\right|(2-\beta)(3-2 \beta)+15 b_{1}^{2}(4-3 \beta)\right]^{2}}{768(2-\beta)^{2}(3-2 \beta)^{2}(4-3 \beta) b_{1}}>0 .
$$

Since $h(t)<\bar{h}(t)$ for all $t \in(0,2)$, we can write

$$
\min \{h(t): t \in(0,2)\} \leq \min \{\bar{h}(t): t \in(0,2)\} .
$$

Now, we will investigate minimum of the function $\bar{h}(t)$ on the open interval $(0,2)$.

Differentiating both sides of 2.31, we have

$$
\bar{h}^{\prime}(t)=\left(3 h_{1} t+2 \bar{h}_{2}\right) t, t \in(0,2) .
$$

Since $h_{1}>0, \bar{h}_{2}>0$, the function $\bar{h}(t)$ is a strictly increasing function on $(0,2)$. Therefore,

$$
\min \{\bar{h}(t): t \in(0,2)\}=\bar{h}(0+)=\lim _{t \rightarrow 0+} \bar{h}(t)=\frac{b_{1}}{3(4-3 \beta)} .
$$


Finally, let $t=2$. In this case the function $G(\xi, \eta, 2)$ is a constant as follows:

$$
G_{2}(\xi, \eta)=G(\xi, \eta, 2)=d_{3}(2)=\frac{\left|b_{1}^{3} \varphi(\beta)-6(2-\beta)^{3} \Lambda\right|+6(2-\beta)^{3}\left|2 b_{2}-b_{1}\right|}{18(2-\beta)^{3}(4-3 \beta)} .
$$

Thus, from (2.29)-2.34) and (2.28), we obtain

$$
\left|a_{4}\right| \leq \min \left\{\frac{\left|b_{1}^{3} \varphi(\beta)-6(2-\beta)^{3} \Lambda\right|+6(2-\beta)^{3}\left|2 b_{2}-b_{1}\right|}{18(2-\beta)^{3}(4-3 \beta)}, \frac{b_{1}}{3(4-3 \beta)}\right\} .
$$

With this, the proof of Theorem 2.1 is completed.

The following theorems are direct results of Theorem 2.1 .

Theorem 2.2. Let the function $f(z)$ given by 1.1$)$ be in the class $S_{\Sigma}^{*}(\phi)$, where $\phi$ is an analytic function given by $(1.2)$. Then,

$$
\left|a_{2}\right| \leq b_{1},\left|a_{3}\right| \leq \begin{cases}b_{1}^{2}, & \text { if } b_{1} \leq \frac{1}{2} \\ \frac{b_{1}}{2}, & \text { if } b_{1}>\frac{1}{2}\end{cases}
$$

and

$$
\left|a_{4}\right| \leq \min \left\{\frac{\left|2 b_{1}^{3}-\Lambda\right|+\left|2 b_{2}-b_{1}\right|}{3}, \frac{b_{1}}{3}\right\},
$$

where $\Lambda=\Lambda\left(b_{1}, b_{2}, b_{3}\right)=b_{1}-2 b_{2}+b_{3}$.

Theorem 2.3. Let the function $f(z)$ given by 1.1) be in the class $C_{\Sigma}(\phi)$, where $\phi$ is an analytic function given by 1.2 . Then,

$$
\left|a_{2}\right| \leq \frac{b_{1}}{2},\left|a_{3}\right| \leq\left\{\begin{array}{l}
\frac{b_{1}^{2}}{4}, \text { if } b_{1} \leq \frac{2}{3}, \\
\frac{b_{1}}{6}, \quad \text { if } b_{1}>\frac{2}{3}
\end{array}\right.
$$

and

$$
\left|a_{4}\right| \leq \min \left\{\frac{\left|5 b_{1}^{3}-4 \Lambda\right|+4\left|2 b_{2}-b_{1}\right|}{48}, \frac{b_{1}}{12}\right\},
$$

where $\Lambda=\Lambda\left(b_{1}, b_{2}, b_{3}\right)=b_{1}-2 b_{2}+b_{3}$.

\section{Concluding Remarks}

If the function $\phi(z)$, aforementioned in study, is given by

$$
\phi(z)=\frac{1+a z}{1+b z}=1+(a-b) z-b(a-b) z^{2}+b^{2}(a-b) z^{3}+\cdots \quad(-1 \leq b<a \leq 1),
$$

then $b_{1}=(a-b), b_{2}=-b(a-b)$ and $b_{3}=b^{2}(a-b)$. 
Taking $a=1-2 \alpha, b=-1$ in 3.1 , we have

$\phi(z)=\frac{1+(1-2 \alpha) z}{1-z}=1+2(1-\alpha) z+2(1-\alpha) z^{2}+2(1-\alpha) z^{3}+\cdots \quad(0 \leq \alpha<1)$.

Hence, $b_{1}=b_{2}=b_{3}=2(1-\alpha)$.

Choosing $\phi(z)$ of the form (3.1) and (3.2) in Theorem 2.1 we can readily deduce the following results, respectively.

Corollary 3.1. Let the function $f(z)$ given by 1.1 be in the class $M_{\Sigma}\left(\frac{1+a z}{1+b z}, \beta\right)$ $(-1 \leq b<a \leq 1,0 \leq \beta \leq 1)$. Then, $\left|a_{2}\right| \leq \frac{a-b}{2-\beta},\left|a_{3}\right| \leq\left\{\begin{array}{ll}\frac{(a-b)^{2}}{(2-\beta)^{2}}, & \text { if } a-b \leq \frac{(2-\beta)^{2}}{2(3-2 \beta)}, \\ \frac{a-b}{2(3-2 \beta)}, & \text { if } a-b>\frac{(2-\beta)^{2}}{2(3-2 \beta)}\end{array} \quad\right.$ and $\left|a_{4}\right| \leq \frac{a-b}{3(4-3 \beta)}$.

Corollary 3.2. Let the function $f(z)$ given by 1.1] be in the class $M_{\Sigma}\left(\frac{1+(1-2 \alpha) z}{1-z}, \beta\right)$ $=M_{\Sigma}(\alpha, \beta), \alpha \in[0,1), \beta \in[0,1]$. Then, $\left|a_{2}\right| \leq \frac{2(1-\alpha)}{2-\beta},\left|a_{3}\right| \leq\left\{\begin{array}{ll}\frac{1-\alpha}{3-2 \beta}, & \text { if } 0 \leq \alpha<1-\alpha_{0}, \\ \frac{4(1-\alpha)^{2}}{(2-\beta)^{2}}, & \text { if } 1-\alpha_{0} \leq \alpha<1,\end{array}\right.$ and $\left|a_{4}\right| \leq \frac{2(1-\alpha)}{3(4-3 \beta)}$, where $\alpha_{0}=\frac{(2-\beta)^{2}}{4(3-2 \beta)}$.

Also, taking $\alpha=0$ in $(3.2)$, we get

$$
\phi(z)=\frac{1+z}{1-z}=1+2 z+2 z^{2}+2 z^{3}+\cdots .
$$

Hence, $b_{1}=b_{2}=b_{3}=2$.

Choosing $\phi(z)$ of the form 3.3 in Theorem 2.1. we arrive at the following corollary.

Corollary 3.3. Let the function $f(z)$ given by 1.1 be in the class $M_{\Sigma}\left(\frac{1+z}{1-z}, \beta\right), \beta \in$ $[0,1]$. Then, $\left|a_{2}\right| \leq \frac{2}{2-\beta},\left|a_{3}\right| \leq \frac{1}{3-2 \beta}$ and $\left|a_{4}\right| \leq \frac{2}{3(4-3 \beta)}$.

Choosing $\phi(z)$ of the form 3.1 and 3.2 in Theorem 2.2 we can readily deduce the following results, respectively.

Corollary 3.4. Let the function $f(z)$ given by 1.1 be in the class $S_{\Sigma}^{*}\left(\frac{1+a z}{1+b z}\right)$ $(-1 \leq b<a \leq 1)$. Then,

$\left|a_{2}\right| \leq a-b,\left|a_{3}\right| \leq\left\{\begin{array}{ll}(a-b)^{2}, & \text { if } a-b \leq \frac{1}{2}, \\ \frac{a-b}{2}, & \text { if } a-b>\frac{1}{2}\end{array} \quad\right.$ and $\left|a_{4}\right| \leq \frac{a-b}{3}$.

Corollary 3.5. Let the function $f(z)$ given by 1.1 be in the class $S_{\Sigma}^{*}\left(\frac{1+(1-2 \alpha) z}{1-z}\right)$ $=S_{\Sigma}^{*}(\alpha), \alpha \in[0,1)$. Then, $\left|a_{2}\right| \leq 2(1-\alpha),\left|a_{3}\right| \leq\left\{\begin{array}{ll}1-\alpha, & \text { if } 0 \leq \alpha<\frac{3}{4}, \\ 4(1-\alpha)^{2}, & \text { if } \frac{3}{4} \leq \alpha<1\end{array} \quad\right.$ and $\left|a_{4}\right| \leq \frac{2(1-\alpha)}{3}$. 
Remark 3.6. In the special case, we can also obtain Corollary 3.4 from Corollary 3.1 and Corollary 3.5 from Corollary 3.2 for $\beta=1$.

Moreover, taking, for example, $\alpha=\frac{3}{4}$ in 3.2 , we have

$$
\phi(z)=\frac{2-z}{2(1-z)}=1+\frac{1}{2} z+\frac{1}{2} z^{2}+\frac{1}{2} z^{3}+\cdots .
$$

Hence, $b_{1}=b_{2}=b_{3}=\frac{1}{2}$.

Choosing $\phi(z)$ of the form (3.4) in Theorem 2.2. we arrive at the following corollary.

Corollary 3.7. Let the function $f(z)$ given by 1.1 be in the class $S_{\Sigma}^{*}\left(\frac{2-z}{2(1-z)}\right)$. Then, $\left|a_{2}\right| \leq \frac{1}{2},\left|a_{3}\right| \leq \frac{1}{4}$ and $\left|a_{4}\right| \leq \frac{1}{6}$.

Remark 3.8. In the special case, we can also obtain Corollary 3.7 from Corollary 3.5 for $\alpha=\frac{3}{4}$.

Choosing $\phi(z)$ of the form (3.1) and $(3.2)$ in Theorem 2.3, we can readily deduce the following results, respectively.

Corollary 3.9. Let the function $f(z)$ given by 1.1) be in the class $C_{\Sigma}\left(\frac{1+a z}{1+b z}\right)$ $(-1 \leq b<a \leq 1)$. Then,

$\left|a_{2}\right| \leq \frac{a-b}{2},\left|a_{3}\right| \leq\left\{\begin{array}{ll}\frac{(a-b)^{2}}{4}, & \text { if } a-b \leq \frac{2}{3}, \\ \frac{a-b}{6}, & \text { if } a-b>\frac{2}{3}\end{array} \quad\right.$ and $\left|a_{4}\right| \leq \frac{a-b}{12}$.

Corollary 3.10. Let the function $f(z)$ given by 1.1 be in the class $C_{\Sigma}\left(\frac{1+(1-2 \alpha) z}{1-z}\right)$ $=C_{\Sigma}(\alpha), \alpha \in[0,1)$. Then, $\left|a_{2}\right| \leq 1-\alpha,\left|a_{3}\right| \leq\left\{\begin{array}{ll}\frac{1-\alpha}{3}, & \text { if } 0 \leq \alpha<\frac{2}{3}, \\ (1-\alpha)^{2}, & \text { if } \frac{2}{3} \leq \alpha<1\end{array}\right.$ and $\left|a_{4}\right| \leq \frac{1-\alpha}{6}$.

Moreover, taking, for example, $\alpha=\frac{2}{3}$ in $(3.2$, we get

$$
\phi(z)=\frac{3-z}{3(1-z)}=1+\frac{2}{3} z+\frac{2}{3} z^{2}+\frac{2}{3} z^{3}+\cdots .
$$

Hence, $b_{1}=b_{2}=b_{3}=\frac{2}{3}$.

Choosing $\phi(z)$ of the form 3.5 in Theorem 2.3. we arrive at the following corollary.

Corollary 3.11. Let the function $f(z)$ given by 1.1 be in the $\operatorname{class} C_{\Sigma}\left(\frac{3-z}{3(1-z)}\right)$. Then,

$\left|a_{2}\right| \leq \frac{1}{3},\left|a_{3}\right| \leq \frac{1}{9}$ and $\left|a_{4}\right| \leq \frac{1}{18}$.

Remark 3.12. In the special case, we can also obtain Corollary 3.11 from Corollary 3.10 for $\alpha=\frac{2}{3}$. 


\section{REFERENCES}

[1] Ali R. M., Lee S. K., Ravichandran V. and Supramanian S., Coefficient estimates for biunivalent Ma-Minda starlike and convex functions, Appl. Math. Lett. 25(3), (2012), 334-351.

[2] Brannan D. A. and Clunie J. G., Aspects of Contemporary Complex Analysis, Proceeding of the NATO Advanced Study Institute held at the University of Durham, Durham; July 1-20, 1979, (Academic Press, New York and London, 1980).

[3] Brannan D. A. and Taha T. S., On some classes of bi-univalent functions, Mathematical Analysis and Its Applications 3, (1985), 18-21.

[4] Bulut S., Coefficient estimates for a class of analytic and bi-univalent functions, Novi Sad J. Mat. 43(2), 59-65, 2013.

[5] Çağlar M., Orhan H. and Yağmur N., Coefficient bounds for new subclasses of bi-univalent functions, Filomat 27(7), 1165-1171, 2013.

[6] Deniz E., Certain subclasses of bi-univalent functions satisfying subordinate conditions, $J$. Classical Anal. 2(1), (2013), 49-60.

[7] Frasin B. A. and Aouf M. K., New subclasses of bi-univalent functions, Appl. Math. Lett. 24(9), (2011), 1569-1573.

[8] Goyal S. P. and Goswami P., Estimate for initial Maclaurin coefficients of bi-univalent functions for a class defined by fractional derivatives, J. Egyptian Math. Soc. 20, (2012), 179-182.

[9] Hayami T. and Owa S., Coefficient bounds for bi-univalent functions, Pan Amer. Math. J. $22(4), 15-26,2012$.

[10] Kim Y. C. and Srivastava H. M., Some subordination properties for spirallike functions, Appl. Math. Comput. 203(2), (2008), 838-842.

[11] Lewin M., On a coefficient problem for bi-univalent functions, Proc. Amer. Math. Soc. 18, (1967), 63-68.

[12] Ma W. C. and Minda D., A unified treatment of some special classes of functions, in: Proceedings of the Conference on Complex Analysis, Tianjin, , (1992),157-169, Conf. Proc. Lecture Notes anal. I, Int. Press, Cambridge, MA, 1994.

[13] Magesh N. and Yamini J., Coeffcient bounds for certain subclasses of bi-univalent functions, Internat. Math. Forum 8(27), (2013), 1337-1344.

[14] Miller S. S. and Mocanu P. T., Differential subordinations, Monographs and Textbooks in Pure and Applied Mathematics 225, Dekker, New York, 2000.

[15] Murugusundaramoorthy G., Magesh N. and Prameela V., Coefficient bounds for certain subclasses of bi-univalent functions, Abs. Appl. Anal., (2013), Article Id 573017, 3 pages.

[16] Netanyahu E., The minimal distance of the image boundary from the origin and the second coefficient of a univalent function in $|z|<1$, Arch. Rational Mech. Anal. 32, (1969), 100-112.

[17] Orhan H., Magesh N. and Balaji V. K., Initial Coefficient Bounds for a General Class of Bi-Univalent Functions, Filomat 29(6), (2015), 1259-1267.

[18] Pommerenke C., Univalent Functions, Vandenhoeck and Rupercht, Göttingen, 1975.

[19] Prema S. and Keerthi B. S., Coefficient bounds for certain subclasses of analytic functions, J. Math. Anal. 4(1), (2013), 22-27.

[20] Ravichandran V., Polatoglu Y., Bolcal M. and Sen A., Certain subclasses of starlike and convex functions of complex order, Hacettepe J. Math. Stat. 34, (2005), 9-15.

[21] Sivaprasad Kumar S., Kumar V. and Ravichandran V., Estimates for the initial coefficients of bi-univalent functions, (2012), arXiv:1203.5480v1.

[22] Srivastava H. M., Mishra A. K. and Gochhayat P., Certain subclasses of analytic and biunivalent functions, Appl. Math. Lett. 23, (2010), 1188-1192.

[23] Srivastava H. M., Some inequalities and other results associated with certain subclasses of univalent and bi-univalent analytic functions, in Nonlinear Analysis: Stability; Approximation; and Inequalities (Panos M. Pardalos, Pando G. Georgiev, and Hari M. Srivastava, Editors), 
Springer Series on Optimization and Its Applications Vol. 68, Springer-Verlag, Berlin, Heidelberg and New York, (2012), 607-630.

[24] Srivastava H. M., Bulut S., Çağlar M. and Yağmur N., Coefficient estimates for a general subclass of analytic and bi-univalent functions, Filomat 27(5), (2013), 831-842.

[25] Srivastava H. M., Murugusundaramoorthy G. and Magesh N., On certain subclasses of biunivalent functions associated with Hohlov operator, Global J. Math. Anal. 1(2), (2013), $67-73$.

[26] Srivastava H. M., Xu Q. H. and Wu G. P., Coefficient estimates for certain subclasses of spiral-like functions of complex order, Appl. Math. Lett. 23(7), (2010), 763-768.

[27] Xu Q. H., Gui Y. C. and Srivastava H. M., Coefficient estimates for a certain subclass of analytic and bi-univalent functions, Appl. Math. Lett. 25(6), (2012), 990-994.

[28] Xu Q. H., Xiao H. G. and Srivastava H. M., A certain general subclass of analytic and bi-univalent functions and associated coefficient estimate problems, Appl. Math. Comput. 218(23), (2012), 11461-11465.

[29] Xu Q. H., Cai Q. M. and Srivastava H. M., Sharp coefficient estimates for certain subclasses of starlike functions of complex order, Appl. Math. Comput. 225, (2013), 43-49.

Current address: Nizami Mustafa: Department of Mathematics, Faculty of Science and Letters, Kafkas University, Kars, 36100, Turkey.

E-mail address: nizamimustafa@gmail.com

ORCID Address: http://orcid.org/0000-0001-9640-8526

Current address: Veysel Nezir (Corresponding author): Department of Mathematics, Faculty of Science and Letters, Kafkas University, Kars, 36100, Turkey.

E-mail address: veyselnezir@yahoo.com

ORCID Address: http://orcid.org/0000-0002-2758-0274 